\title{
Antibiotic stability over six weeks in aqueous solution at body temperature with and without heat treatment that mimics the curing of bone cement
}

\section{E. Samara, T. F. Moriarty, L. A. Decosterd, R. G. Richards, E. Gautier, P. Wahl}

AO Research Institute Davos, Davos, Switzerland
E. Samara, MD, Research

fellow,

- T. F. Moriarty, PhD, Principal Scientist,

R. G. Richards, PhD, Professor, Director, AO Research Institute Davos, Davos, Switzerland.

- L. A. Decosterd, PhD, Professor, Head of the Laboratory of Clinical

Pharmacology, University Hospital

Lausanne and University of

Lausanne, Lausanne, Switzerland.

- E. Gautier, MD, Professor, Head of the Department of Orthopaedic Surgery, HFR Fribourg - Cantonal Hospital, Fribourg, Switzerland.

P. Wahl, MD, Consultant,

Division for Orthopaedic and

Trauma Surgery, Cantonal

Hospital Winterthur, Winterthur,

Switzerland.

Correspondence should be sent to T. F. Moriarty; email:

fintan.moriarty@aofoundation.org

doi: $10.1302 / 2046-3758.65 . B J R-$ 2017-0276.R1

Bone Joint Res 2017;6:296-306.

Received: 14 October 2016;

Accepted: 27 February 2017

\section{Objectives}

Thermal stability is a key property in determining the suitability of an antibiotic agent for local application in the treatment of orthopaedic infections. Despite the fact that long-term therapy is a stated goal of novel local delivery carriers, data describing thermal stability over a long period are scarce, and studies that avoid interference from specific carrier materials are absent from the orthopaedic literature.

\section{Methods}

In this study, a total of 38 frequently used antibiotic agents were maintained at $37^{\circ} \mathrm{C}$ in saline solution, and degradation and antibacterial activity assessed over six weeks. The impact of an initial supplementary heat exposure mimicking exothermically curing bone cement was also tested as this material is commonly used as a local delivery vehicle. Antibiotic degradation was assessed by liquid chromatography coupled to mass spectrometry, or by immunoassays, as appropriate. Antibacterial activity over time was determined by the Kirby-Bauer disk diffusion assay.

\section{Results}

The heat exposure mimicking curing bone cement had minimal effect on stability for most antibiotics, except for gentamicin which experienced approximately $25 \%$ degradation as measured by immunoassay. Beta-lactam antibiotics were found to degrade quite rapidly at $37^{\circ} \mathrm{C}$ regardless of whether there was an initial heat exposure. Excellent long-term stability was observed for aminoglycosides, glycopeptides, tetracyclines and quinolones under both conditions.

\section{Conclusions}

This study provides a valuable dataset for orthopaedic surgeons considering local application of antibiotics, and for material scientists looking to develop next-generation controlled or extended-release antibiotic carriers.

Cite this article: Bone Joint J 2017;6:296-306

Keywords: Local antibiotics, Thermal stability, Bone cement

\section{Article focus}

- Comprehensive data describing thermal stability of antibiotic agents in aqueous solution at physiological temperature for local application in orthopaedic indications are not available.

- Thermal stability at $37^{\circ} \mathrm{C}$ of 37 antibiotic drugs over six weeks is described.

- As bone cement is frequently used in orthopaedic surgery for the local delivery of antibiotics, the potential effect of the exothermic curing process of bone cement on antibiotic agents was also examined.

\section{Key messages}

- Aminoglycosides, glycopeptides, tetracyclines and quinolones have good thermal stability over six weeks, but local toxicity issues might limit local application of tetracyclines and quinolones.

- While beta-lactams show exponential degradation over time, sustained antibacterial activity can be observed for up to 
three weeks, with aztreonam displaying even greater stability.

- The heat generated by curing bone cement does not have any effect on the majority of the antibiotic agents tested, except for gentamicin, that degraded by approximately $25 \%$.

\section{Strengths and limitations}

- Two analytical methods have been used: qualitative assessment of antimicrobial activity by disk diffusion tests; and quantification by liquid chromatography coupled to mass spectrometry, or fluorescence polarisation immunoassays, as available.

- Degradation half-lives have been calculated from quantification data, but these methods were available for only 20 antibiotics.

- Antibacterial activity has been tested by the KirbyBauer disk diffusion assay, but interpretation of inhibition zone results has limitations, particularly as some substances have inconstant diffusion or degradation on agar.

\section{Introduction}

Local delivery of antibiotics enables high drug concentrations to be achieved at the desired site of antimicrobial action, while keeping systemic exposure and toxicity low. ${ }^{1-3}$ The following characteristics are desirable for a given antimicrobial agent to be suitable for local application: adequate antimicrobial spectrum; chemical compatibility with, and adequate release kinetics from the chosen carrier; appropriate solubility; low local toxicity; low systemic exposure and/or toxicity; and low risk of hypersensitivity. ${ }^{1-5}$ Thermal stability is a key factor in determining an antibiotic's suitability for local application, as it must not only resist any heat generated during the manufacturing process of the carrier, but also be stable at body temperature over the entire duration of the release period inside the patient.2,4 Despite the growing use of locally delivered antimicrobial agents, data regarding thermal stability in such settings are incomplete, scarce or even lacking entirely.

A large number of studies have been published that examine drug release in vitro and in vivo from various carrier materials. ${ }^{3,5-9}$ However, these studies primarily tested compatibility with carrier substances and were designed to quantify the amount of antibiotic released from the matrix. The most commonly tested condition is antibiotic release from bone cement (polymethyl methacrylate, PMMA), which has been shown to release antibiotic over a number of weeks. ${ }^{6}$ However, such data are specific to the formulation of the bone cement and do not translate to other materials. Overall, the limited data available at present have studied antibiotic stability over a short period of time and below body temperature or with a restricted range of antibiotics. ${ }^{10}$ Such studies have generally been performed with the purpose of testing portable infusion devices for prolonged intravenous antibiotic therapy in an outpatient setting, for continuous antibiotic infusion to optimise antimicrobial pharmacodynamics in critical care, or for the use of antibiotics in peritoneal dialysis solution. ${ }^{11-16}$ Information regarding thermal stability of antibiotics exposed to body temperature over a prolonged period is thus necessary for a comprehensive assessment of the suitability of their use for local application.

The primary aim of this study was therefore to examine the stability of solutions of 38 antibiotics maintained at $37^{\circ} \mathrm{C}$ for six weeks, a common duration of treatment in the orthopaedic setting. ${ }^{17,18}$ Such data are critical for any future controlled extended release antibiotic delivery vehicle. As PMMA remains the most frequently used carrier material in orthopaedic applications, ${ }^{3-5}$ the secondary objective of this study was to examine the impact of an initial thermal treatment simulating the exothermic process of PMMA polymerisation, followed by exposure to $37^{\circ} \mathrm{C}$ for six weeks, on antibiotic stability. This study will therefore provide an overview of the thermal stability of a broad range of antibiotics, helping to guide clinicians in selecting the most appropriate agents for local application.

\section{Materials and Methods}

Antibiotic agents. Antibiotic agents were selected on the basis of clinical experience in local application, published experimental data, and potential for local application based on chemical properties and antimicrobial mode of action. Preference was given to clinically available antibiotic preparations, rather than analytical or research preparations. Solutions were used only if no powdered form was available. A total of 38 antibiotic agents were included, five of which were combination products. The combination products involved either two partner antibiotics or one antibiotic with an enzyme inhibitor. In the case of combination products, only the antibiotic agent(s) for which an analytical methodology was available were measured. A comprehensive list is provided in Table I. Each antibiotic was dissolved or diluted in $0.9 \%$

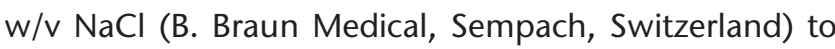
an arbitrary initial target concentration of $200 \mathrm{mg} / \mathrm{l}$. The prepared solutions, therefore, contained the antibiotic drug as well as any excipients present in the pharmaceutical formulations. Details of preservatives and excipients present in the antibiotic may be found, where present, on the package inserts. Antibiotics were generally available as salt, and less frequently as base or acid. Each antibiotic solution was divided into two equal portions immediately after preparation. One portion was directly incubated at $37^{\circ} \mathrm{C}$ for 42 days (Regular group) and the second exposed to a thermal curve mimicking the exothermic bone cement curing process (as described 
Table I. List of tested antibiotics and corresponding methods for measuring drug concentration and antimicrobial activity

\begin{tabular}{|c|c|c|c|}
\hline Antibiotic & Brand name (Supplier) & Quantification & Disk Diffusion \\
\hline \multicolumn{4}{|l|}{ Penicillins } \\
\hline Amoxicillin & Clamoxyl (GlaxoSmithKline, Münchenbuchsee, Switzerland) & \multirow[t]{2}{*}{ LC-MS/MS } & S. aureus NCTC 12973 \\
\hline Amoxicillin+clavulanate & Co-Amoxi-Mepha (Mepha Pharma, Basel, Switzerland) & & S. aureus NCTC 12973 \\
\hline Flucloxacillin & Floxapen (Actavis, Regensdorf, Switzerland) & \multirow[t]{3}{*}{ LC-MS/MS } & S. aureus NCTC 12973 \\
\hline Ticarcillin+clavulanate & Timentin (GlaxoSmithKline, Brentford, United Kingdom) & & S. aureus NCTC 12973 \\
\hline Temocillin & Negaban (Eumedica, Manage, Belgium) & & E. coli NCTC 12241 \\
\hline Piperacillin+tazobactam & $\begin{array}{l}\text { Piperacillin Tazobactam (Sandoz Pharmaceuticals, Risch-Rotkreuz, } \\
\text { Switzerland) }\end{array}$ & LC-MS/MS & S. aureus NCTC 12973 \\
\hline \multicolumn{4}{|l|}{ Cephalosporins } \\
\hline Cefazolin & Kefzol (Teva Pharma, Basel, Switzerland) & LC-MS/MS & S. aureus NCTC 12973 \\
\hline Cefuroxime & Zinacef (GlaxoSmithKline, Münchenbuchsee, Switzerland) & \multirow{2}{*}{ LC-MS/MS } & S. aureus NCTC 12973 \\
\hline Cefotaxime & Claforan (Sanofi-Aventis, Vernier, Switzerland) & & S. aureus NCTC 12973 \\
\hline Ceftriaxone & Rocephin (Roche Pharma, Reinach, Switzerland) & LC-MS/MS & S. aureus NCTC 12973 \\
\hline Ceftazidime & Fortam (GlaxoSmithKline, Münchenbuchsee, Switzerland) & LC-MS/MS & S. aureus NCTC 12973 \\
\hline Cefepime & Cefepime OrPha (OrPha Swiss, Küsnacht, Switzerland) & LC-MS/MS & S. aureus NCTC 12973 \\
\hline \multicolumn{4}{|c|}{ Carbapenems and monobactams } \\
\hline Imipenem+cilastatin & Imipenem-Cilastatin Labatec (Labatec Pharma, Meyrin, Switzerland) & LC-MS/MS & S. aureus NCTC 12973 \\
\hline Meropenem & Meropenem Labatec (Labatec Pharma, Meyrin, Switzerland) & LC-MS/MS & S. aureus NCTC 12973 \\
\hline Ertapenem & Invanz (Merck \& Co, Lucerne, Switzerland) & \multirow[t]{2}{*}{ LC-MS/MS } & S. aureus NCTC 12973 \\
\hline Aztreonam & Azactam (Bristol-Myers Squibb, Cham, Switzerland) & & E. coli NCTC 12241 \\
\hline \multicolumn{4}{|l|}{ Glycopeptides and lipopeptides } \\
\hline Vancomycin & Vancocin (Teva Pharma, Basel, Switzerland) & \multirow[t]{3}{*}{ LC-MS/MS } & S. aureus NCTC 12973 \\
\hline Teicoplanin & Teicoplanin Labatec (Labatec Pharma, Meyrin, Switzerland) & & S. aureus NCTC 12973 \\
\hline Telavancin & Vibativ (Theravance, San Francisco, California) & & S. aureus NCTC 12973 \\
\hline Daptomycin & Cubicin (Novartis Pharma, Basel, Switzerland) & LC-MS/MS & S. aureus NCTC 12973 \\
\hline \multicolumn{4}{|l|}{ Aminoglycosides } \\
\hline Amikacin & Amikin (Bristol-Myers Squibb, Cham, Switzerland) & FPIA & S. aureus NCTC 12973 \\
\hline Gentamicin & Gentamicin-MP (InfectoPharm, Heppenheim, Germany) & FPIA & S. aureus NCTC 12973 \\
\hline Tobramycin & Obracin (Teva Pharma, Basel, Switzerland) & FPIA & S. aureus NCTC 12973 \\
\hline \multicolumn{4}{|l|}{ Quinolones } \\
\hline Ciprofloxacin & Ciproxine (Bayer, Zürich, Switzerland) & LC-MS/MS & S. aureus NCTC 12973 \\
\hline Levofloxacin & Tavanic (Sanofi-Aventis, Vernier, Switzerland) & \multirow[t]{2}{*}{ LC-MS/MS } & S. aureus NCTC 12973 \\
\hline Moxifloxacin & Avalox (Bayer, Zürich, Switzerland) & & S. aureus NCTC 12973 \\
\hline \multicolumn{4}{|l|}{ Macrolides and ketolides } \\
\hline Erythromycin & Erythrocine (Pro Concepta, Zug, Switzerland) & & S. aureus NCTC 12973 \\
\hline Clarithromycin & Klacid (Abbott, Baar, Switzerland) & & S. aureus NCTC 12973 \\
\hline Azithromycin & Zithromax (Pfizer, Zürich, Switzerland) & & S. aureus NCTC 12973 \\
\hline Telithromycin & Ketek (Sanofi-Aventis, Vernier, Switzerland) & & S. aureus NCTC 12973 \\
\hline \multicolumn{4}{|l|}{ Tetracyclines and glycylcyclines } \\
\hline Doxycycline & Vibravenös (Pfizer, Zürich, Switzerland) & & S. aureus NCTC 12973 \\
\hline Minocycline & Minac (Spirig Pharma, Egerkingen, Switzerland) & & S. aureus NCTC 12973 \\
\hline \multicolumn{4}{|l|}{ Various } \\
\hline Rifampin & Rimactan (Sandoz Pharmaceuticals, Risch, Switzerland) & LC-MS/MS & S. aureus NCTC 12973 \\
\hline Clindamycin & Clindamycin (Sandoz) & LC-MS/MS & S. aureus NCTC 12973 \\
\hline Linezolid & Zyvoxid (Pfizer, Zürich, Switzerland) & \multirow[t]{3}{*}{ LC-MS/MS } & S. aureus NCTC 12973 \\
\hline Fusidic acid & Fucidin (Leo Pharmaceuticals, Regensdorf-Watt, Switzerland) & & S. aureus NCTC 12973 \\
\hline Trimethoprim with sulfamethoxazole & Bactrim (Roche Pharma, Reinach, Switzerland) & & S. aureus NCTC 12973 \\
\hline Colistimethate & Colistin (Forest Laboratories, Zürich, Switzerland) & LC-MS/MS & P. aeruginosa $\mathrm{PAO} 1$ \\
\hline
\end{tabular}

LC-MS/MS, High-performance liquid chromatography coupled to tandem mass spectrometry; FPIA, fluorescence polarisation immunoassay; DD, disk diffusion; NCTC, national collection of type cultures.

below), followed by incubation at $37^{\circ} \mathrm{C}$ for 42 days (Heat group). Aliquots of the antibiotic solution were taken after preparation, and again after 12 and 24 hours, and at two, three, five, seven, 14, 21 and 42 days. At each time point, triplicate $1 \mathrm{ml}$ aliquots were withdrawn and frozen in cryotubes (Thermo Fisher Scientific (Schweiz), Reinach, Switzerland), and stored at $-80^{\circ} \mathrm{C}$ until analysis. Samples were analysed as a single batch upon completion of the study.

Temperature monitoring and exposure: determination of temperature-time profile during PMMA polymerisation. A $40 \mathrm{~g}$ package of commercially available high viscosity bone cement (Palacos R; Heraeus Medical, Wehrheim,
Germany) was prepared according to manufacturer instructions using manual mixing. The cement dough was then poured into a $60 \mathrm{ml}$ syringe. A thermocouple sensor (Type K Thermocouple; Pico Technology, Eaton Socon, United Kingdom) was inserted $5 \mathrm{~mm}$ into the cement, and the second sensor recorded ambient air temperature. After 12 minutes, when the cement had hardened, the syringe was removed and the cement block, with the thermocouple still in place, was immersed in a water bath at $37^{\circ} \mathrm{C}$ (Sonorex Super; Bandelin electronic, Berlin, Germany) to mimic the positioning of a formed cement spacer in the human body. Cement and water temperature were measured at 15 second intervals (recorded by 
USB TC-08 Thermocouple Data Logger and PicoLog data acquisition, Pico Technology). The experiment was performed in triplicate.

Temperature monitoring and exposure : heat exposure mimicking PMMA polymerisation. A steam autoclave (Vitaris, Münchenstein, Switzerland) was used to subject test solutions to a heat programme that would mimic the observed temperature-time profile of curing cement. Glass bottles containing $150 \mathrm{ml}$ of antibiotic solution were placed in the pre-warmed autoclave. Temperature monitoring in the liquid was performed through a thermocouple sensor directly immersed in $0.9 \% \mathrm{NaCl}$ solution, with measurements recorded every 15 seconds. Once a maximum chamber temperature of $83^{\circ} \mathrm{C}$ had been reached and maintained for 60 seconds, the solutions were removed and exposed to ambient air for three minutes. Subsequently, the bottles were immersed in a water bath at $17^{\circ} \mathrm{C}$. Temperatures were recorded every 15 seconds from commencement of the heat cycle until the temperature of $37^{\circ} \mathrm{C}$ was reached. The water bath was set to $17^{\circ} \mathrm{C}$ in order to accept the heat transfer from the antibiotic solution-containing bottles, and still achieve the target of $37^{\circ} \mathrm{C}$ within a time-frame closely matching curing PMMA. The target chamber temperature of $83^{\circ} \mathrm{C}$ was set so that the solution may exceed this temperature, but still not reach boiling point. The above-described heat cycle has been chosen to follow as closely as possible the heat-time profile of curing PMMA, without causing the test solutions to boil.

Assessment of antibiotic concentration and activity: high performance liquid chromatography coupled to tandem mass spectrometry. The concentrations of 19 antibiotic agents were measured by high-performance liquid chromatography coupled to tandem mass spectrometry (LCMS/MS) using adaptations of validated methods used in therapeutic drug monitoring in patients, or by specifically developing LC-MS/MS assays for this project (Table I). Antibiotic drug concentrations were determined from samples, thawed and analysed as a single batch after appropriate dilution of aliquots, collected at 0 hour and at days one, three, seven and 42. Reverse-phase chromatographic separation of antibiotics was obtained using stepwise gradient elution of the mobile phase, consisting of $10 \mathrm{mM}$ ammonium formate solution with $0.4 \%$ formic acid and acetonitrile delivered onto a $1.8 \mu \mathrm{m}$ UPLC column Waters Acquity UPLC HSS T3 $2.1 \times 50$ mm (Waters Corp., Milford, Massachusetts).

Detection was performed by electrospray ionisationtriple quadrupole mass spectrometry on Quantum mass spectrometers (Thermo Fisher Scientific, Waltham, Massachusetts), by selected reaction monitoring detection, using the specific antibiotic transitions in the positive mode, except for cefuroxime (negative mode, as cefuroxime is negatively charged after ionisation). Antibiotic quantification was performed using matrix-matched calibration samples using the stable isotopically labelled internal standard of each antibiotic (except for vancomycin, for which no labelled internal standard was available and therefore an external calibration was applied). The lower limits of quantification (LOQ) range from $0.02 \mathrm{mg} / \mathrm{l}$ to $1 \mathrm{mg} / \mathrm{l}$. Individual LOQ limits were $0.02 \mathrm{mg} / \mathrm{l}$ for rifampin, $0.05 \mathrm{mg} / \mathrm{l}$ for cefazolin, cefepime, imipenem, meropenem, tazobactam, and colistin, $0.08 \mathrm{mg} / \mathrm{l}$ for piperacillin, $0.1 \mathrm{mg} / \mathrm{l}$ for amoxicillin, flucloxacillin, cefuroxime, ceftazidime, ertapenem, ciprofloxacin, levofloxacin, clindamycin, and linezolid, $0.5 \mathrm{mg} / \mathrm{l}$ for ceftriaxone and daptomycin, and $1 \mathrm{mg} / \mathrm{l}$ for vancomycin.

Assessment of antibiotic concentration and activity: fluorescence polarisation immunoassays. The concentrations of gentamicin, tobramycin and amikacin were measured by fluorescence polarisation immunoassay (FPIA) on a Cobas Integra 400 plus (Roche Diagnostics International, Rotkreuz, Switzerland) using the standard reagents of the commercial kit. Appropriate dilution of test solutions was performed with human plasma since immunoassays can only be performed in the same biological matrix that is used for calibration. The LOQ was $0.5 \mathrm{mg} / \mathrm{l}, 0.74 \mathrm{mg} / \mathrm{l}$ and $2.5 \mathrm{mg} / \mathrm{l}$ for gentamicin, tobramycin and amikacin, respectively.

Assessment of antibiotic concentration and activity: disk diffusion test. Antimicrobial activity of all antibiotic solutions was measured by the Kirby-Bauer disk diffusion method, performed following the European Committee on Antimicrobial Susceptibility Testing (EUCAST) protocol. ${ }^{19,20}$ Reference strains E. coli NCTC 12241, P. aeruginosa PAO1, or S. aureus NCTC 12973, were chosen depending on the spectrum of activity of the antibiotic tested, and are shown in Table I. Samples were tested in triplicate using $7 \mathrm{~mm}$ paper disks (Becton Dickinson, Heidelberg, Germany), impregnated with $20 \mu \mathrm{l}$ sample solution. The disks were placed on circular Petri dishes with $25 \mathrm{ml}$ Mueller Hinton agar (Thermo Fisher Scientific Oxoid Ltd, Basingstoke, United Kingdom), which was inoculated with a suspension of the reference organism. Each test batch included a control disk containing $10 \mu \mathrm{g}$ gentamicin (Becton Dickinson) to ascertain proper preparation and application of the bacterial suspension. Plates were incubated at $37^{\circ} \mathrm{C}$ for 20 hours, at which time zones of inhibition (ZOI) were measured to the nearest whole millimetre.

Data analysis. Mean values and standard deviations have been calculated from triplicate samples, for the results from LC-MS/MS, FPIA, and also disk diffusion assays. The results of LC-MS/MS or FPIA quantifications were expressed as a percentage of the initial concentration, i.e. without the initial heat exposure. A two-way ANOVA was used to compare differences between the regular and body temperature groups for the LC-MS/MS data. Sidak's post hoc test was used to control for multiple comparisons. The results from the disk diffusion tests are 


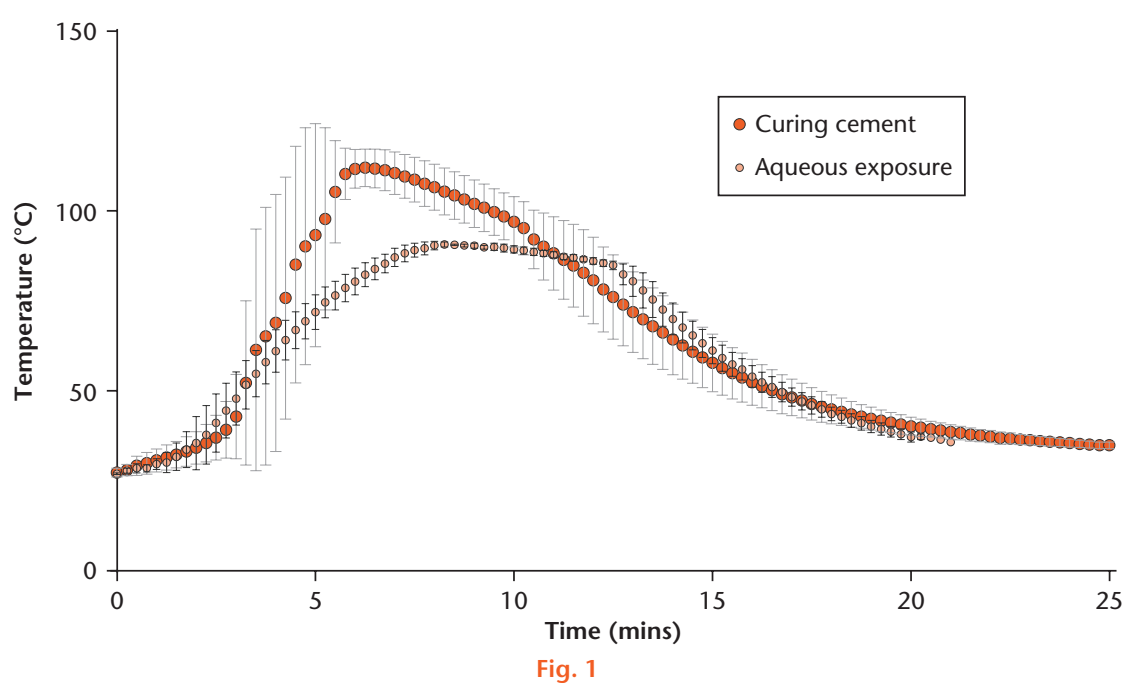

Temperature-time curve of curing polymethyl methacrylate (PMMA) bone cement (large red circle with light grey error bars) and the simulated curve (small circles with black error bars), to which antibiotic solutions were exposed. Measurements were taken every 15 seconds, data represent mean results of a triplicate and error bars represent standard deviation.

indicated as diameter of the zone of inhibition. A twoway ANOVA was used to compare differences between the ZOI diameter over time. In all cases, significance was accepted at $p<0.05$. Prism software was used for all statistical tests (GraphPad Software, La Jolla, California). The in vitro half-lives $\left(\mathrm{t}_{1 / 2}\right)$ of the antibiotics measured using LC-MS/MS were calculated as $\ln 2 / \mathrm{ke}$, considering the terminal slope of the decay, where ke is ( $\ln C_{\text {day }}-$ In $\left.\mathrm{C}_{\text {day } 42}\right) /\left(\mathrm{T}_{\text {day } 42}-\mathrm{T}_{\text {day } 7}\right)$.

\section{Results}

Determination of temperature-time profile during PMMA polymerisation. The temperature-time profiles of curing PMMA and the heat exposure programme applied to the antibiotic solutions are shown in Figure 1. For curing $P M M A$, the time required from initial mixing until equilibration with the $37^{\circ} \mathrm{C}$ water bath was 22 minutes. The highest temperature measured during this time was $111.7^{\circ} \mathrm{C}$. The replication of the temperature-time curve for the antibiotic solutions also lasted 22 minutes, although the peak temperature was lower $\left(90.7^{\circ} \mathrm{C}\right)$ in order to prevent the aqueous solutions from boiling.

Quantification by LC-MS/MS and FPIA. The concentration of the antibiotics determined by LC-MS/MS and FPIA, plotted against time, are summarised in Figure 2 for both the regular and the heat groups. Statistically significant differences between both groups have been marked in Figure 2. Only gentamicin showed a drop in concentration of about $25 \%$ due to the initial heat treatment, but remained stable thereafter. Results for clindamycin have been eliminated because the original antibiotic solution apparently decomposed during storage and no drug or degradation product could be measured.

Assessment of antibacterial activity by disk diffusion test. ZOI sizes determined by the disk diffusion tests are illustrated for all antibiotic agents in Figure 3. Again, clindamycin was inactive, suggestive of drug decomposition during storage, and thus not reported. The difference in ZOI between the regular and the heat groups were usually within $1 \mathrm{~mm}$ to $2 \mathrm{~mm}$, and thus only results from the regular group are illustrated graphically. Statistically significant differences have been marked in the figure, where appropriate. The only antibiotics that showed a significant reduction of ZOI by $3 \mathrm{~mm}$ to $4 \mathrm{~mm}$ in the heat group as compared with the body temperature group were amoxicillin with clavulanate (but not amoxicillin alone), cefazolin, cefuroxime, cefotaxime, ceftriaxone and ertapenem, but these differences were only noticeable on day one. Both curves thereafter were virtually identical. Daptomycin showed a marked variability of ZOI over time, which can be explained in part by the limited reliability of the bioassay method for this antibiotic.

Calculation of degradation half-life in vitro. Degradation half-life values calculated from LC-MS/MS and FPIA data are given in Table II. When the variability in concentrations was within the analytical variability with profiles essentially parallel to the $x$-axis, the antibiotic was considered to be stable and marked as such without indicating calculated values. Since no significant differences have been observed between the regular and the heat groups, calculations have been performed with results from the regular group only.

\section{Discussion}

Thermal stability is a key feature in determining whether a given antibiotic is suitable for local application. 2,4 The present study provides a comprehensive dataset on the thermal stability of 37 antibiotic agents, monitored over six weeks - a duration which may be targeted for future local antibiotic delivery, as it generally corresponds to the standard duration of treatment of bone and implant-associated infections. ${ }^{17,18}$ The potentially deleterious impact on 
Penicillins

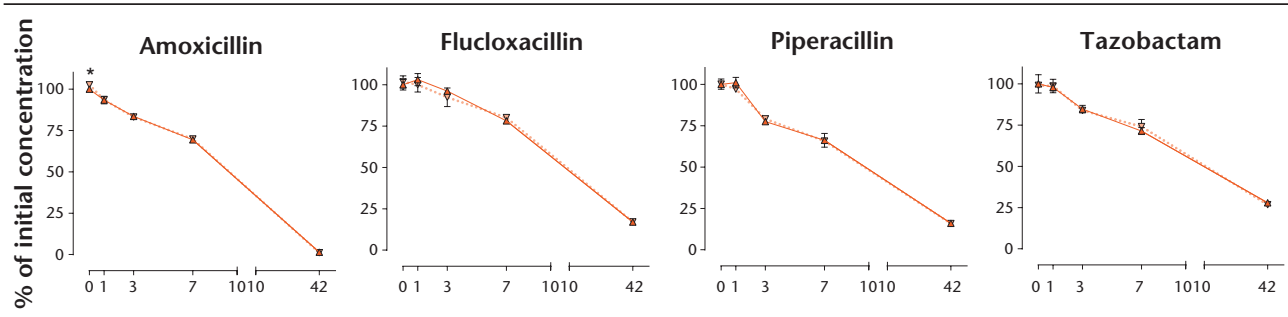

\section{Cephalosporins}
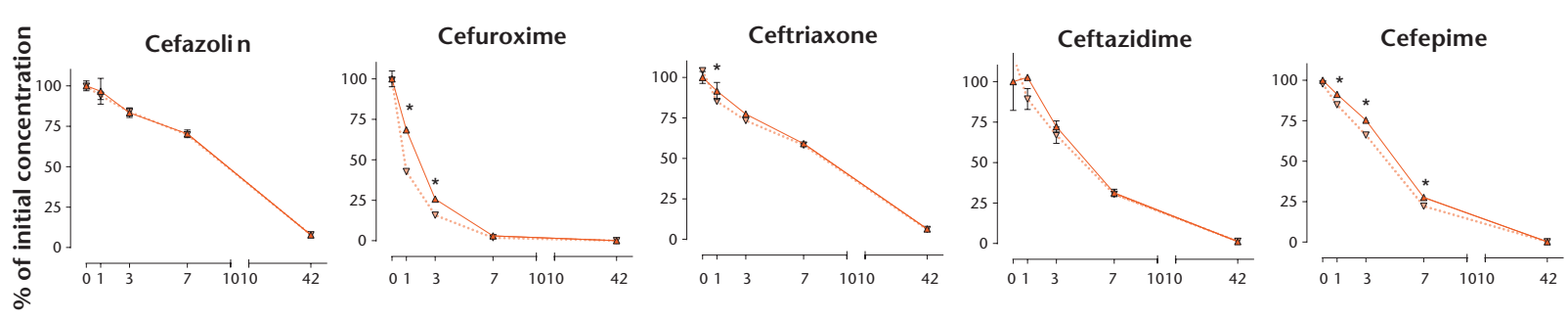

\section{Carbapenems}
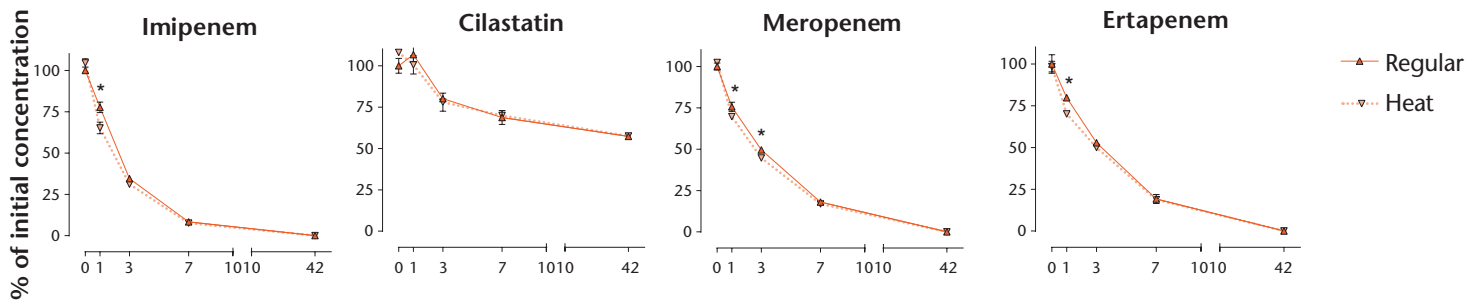

Glycopeptides and Lipopeptides

Aminoglycosides
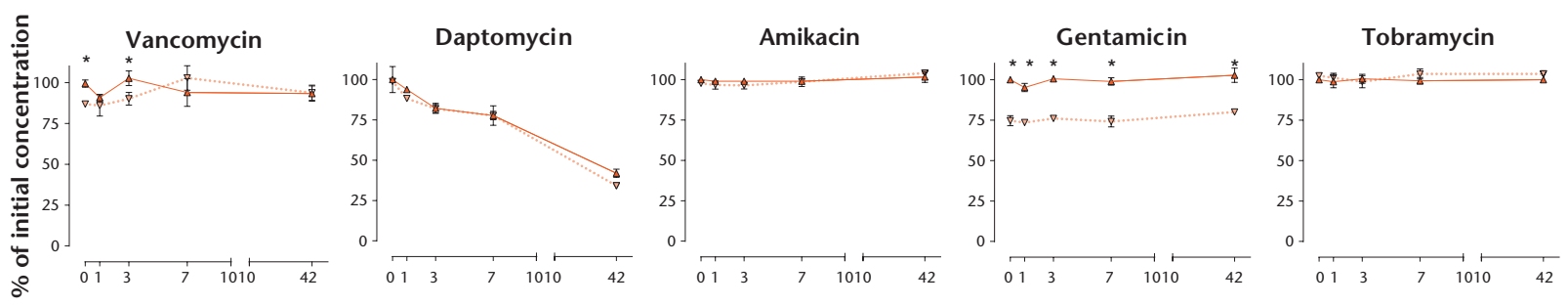

Quinolones

Various
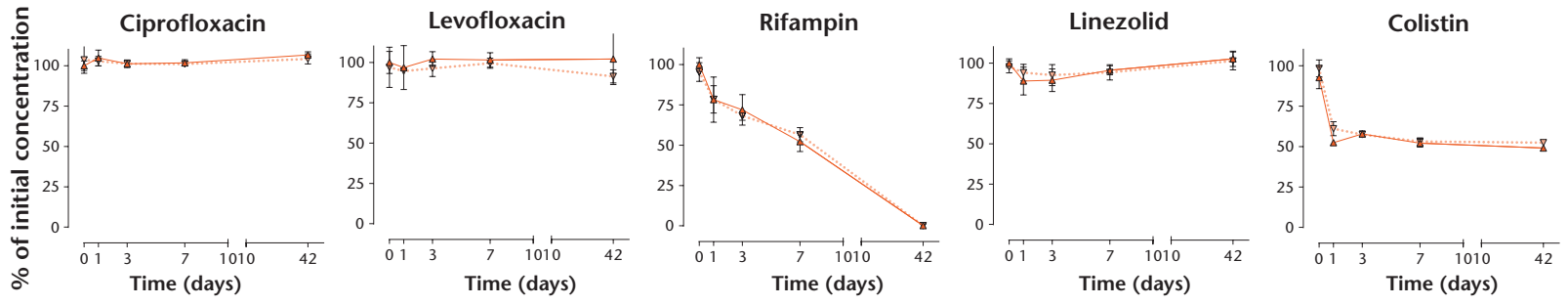

Fig 2

Thermal stability determined for each antibiotic over time as measured by LC-MS/MS or FPIA. Antibiotic solutions were prepared and maintained at $37^{\circ} \mathrm{C}$ for 42 days (regular group, solid lines), or first exposed to a heat profile representative of curing bone cement, followed by exposure to body temperature for 42 days (dashed lines). Concentrations in samples taken prior to heat exposure served as $100 \%$ reference. Data are means of triplicate experiments. Statistically significant differences between measurements from the regular group and the heat exposed group are marked with an asterisk where appropriate.

antibiotic integrity of the initial heat released during the curing of PMMA, the carrier material used most commonly in orthopaedic and trauma surgery, ${ }^{3-5}$ has also been examined as a secondary, additional outcome relevant to current clinical practice. Results from this study were focused on applications in orthopaedics, but may also find 


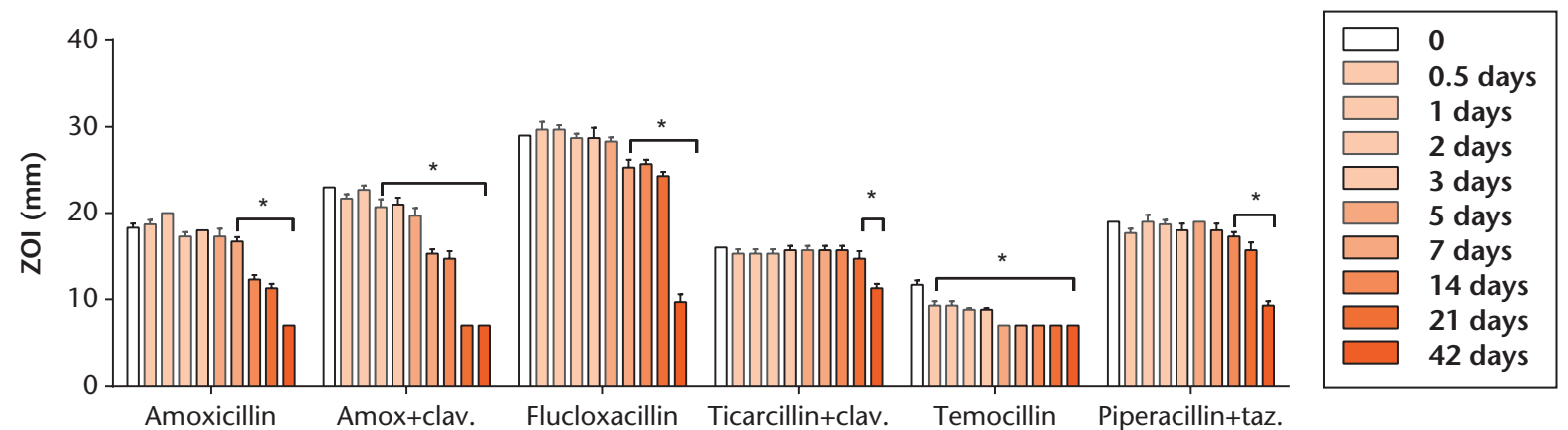

Penicillins
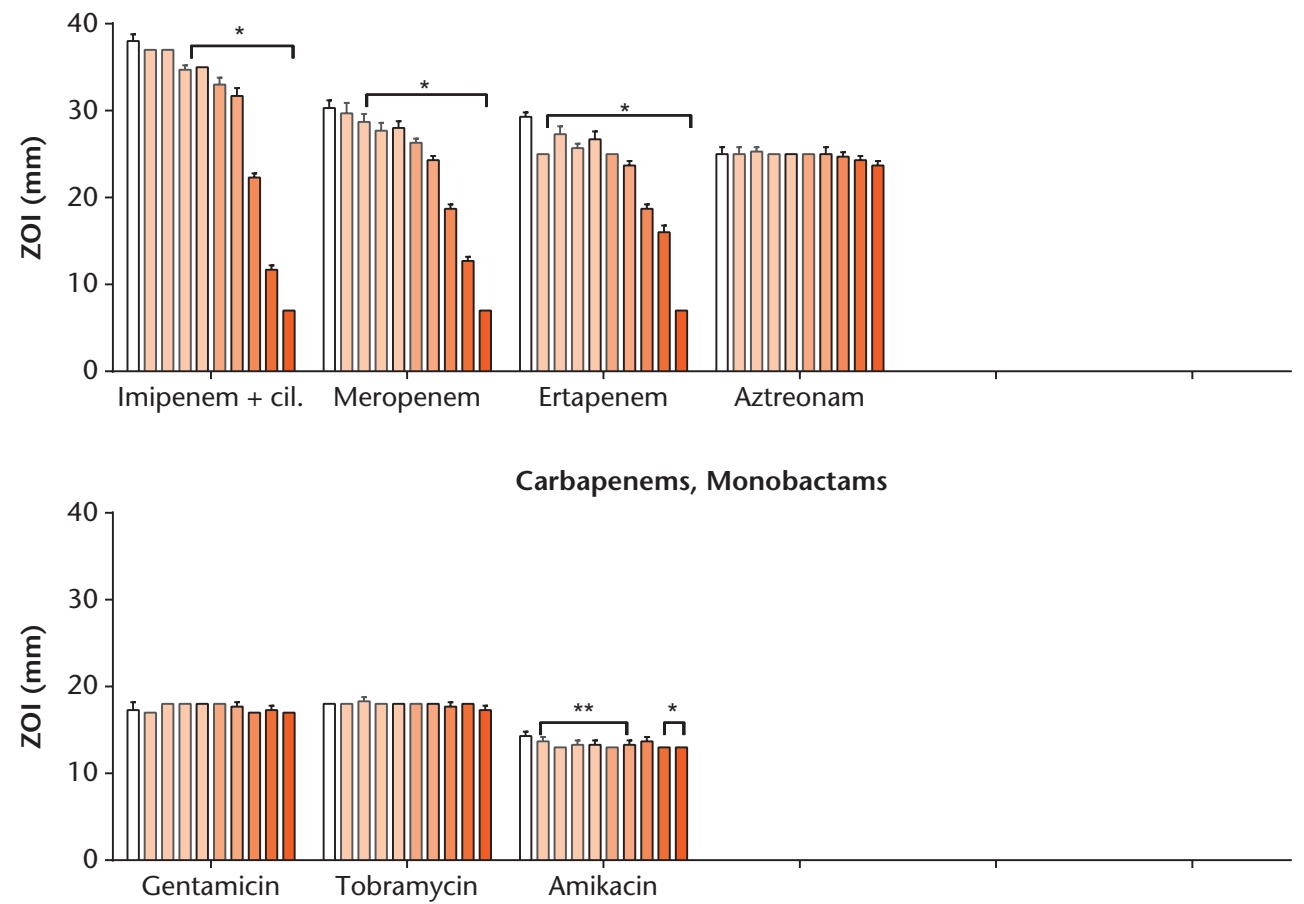

Aminoglycosides

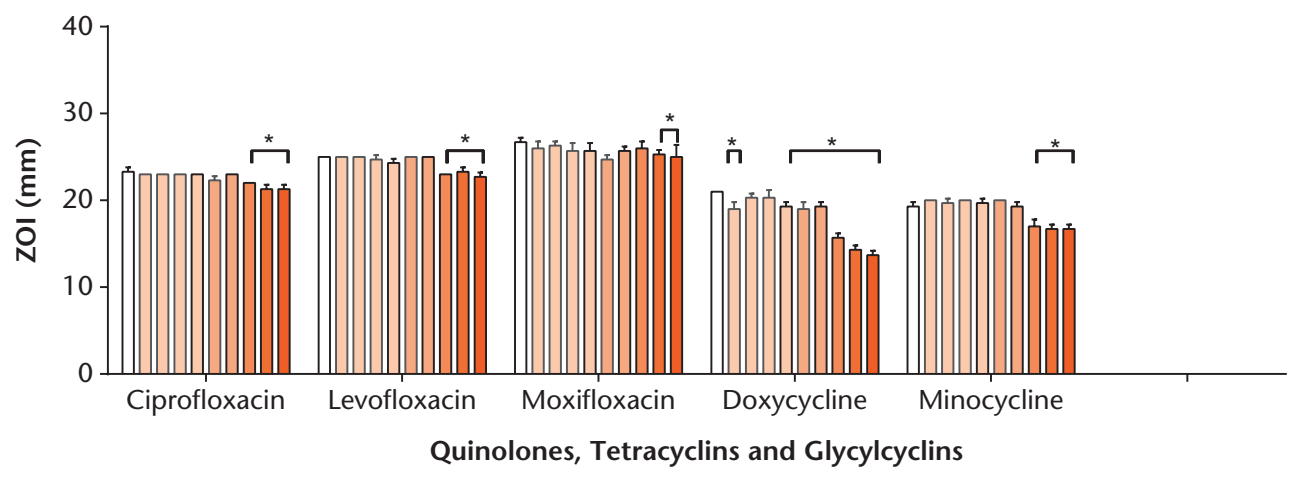

Fig. 3a

Charts showing thermal stability measured for each antibiotic over time, as determined by bioassay. Results are expressed as means from triplicate experiments. Zone of inhibition (ZOI) indicates diameter of the zone of inhibition, in millimetres. Statistically significant variations of the ZOI relative to starting values are marked with an asterisk where appropriate. Antibiotic solutions were prepared and maintained at $37^{\circ} \mathrm{C}$ for 42 days (body temperature group). Of note, temocillin and aztreonam have been tested with E. coli NCTC 12241, and colistimethate with P. aeruginosa PAO1, whereas all other results are from inhibition of S. aureus NCTC 12973. 

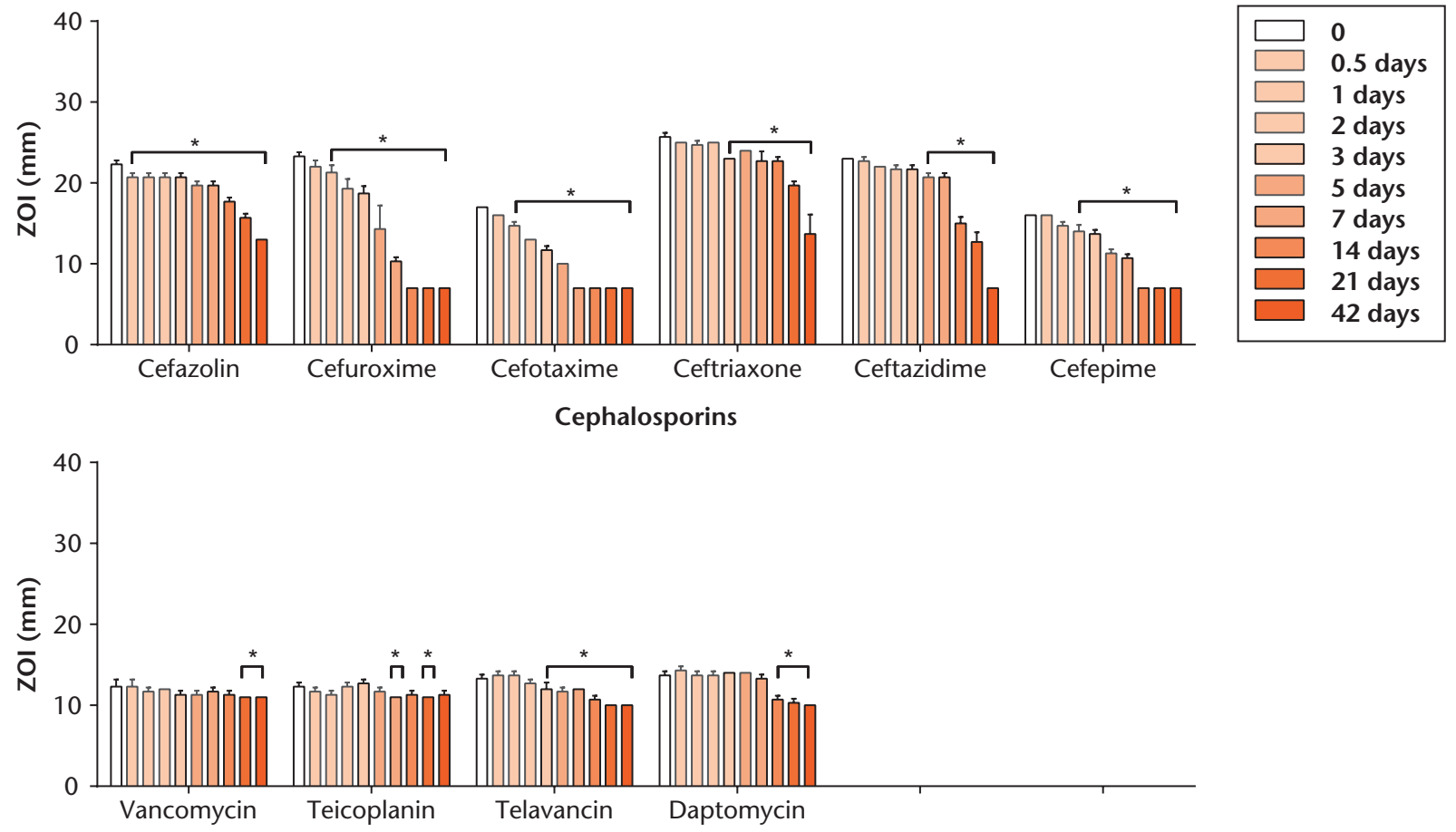

Lipopeptides and Glycopeptides

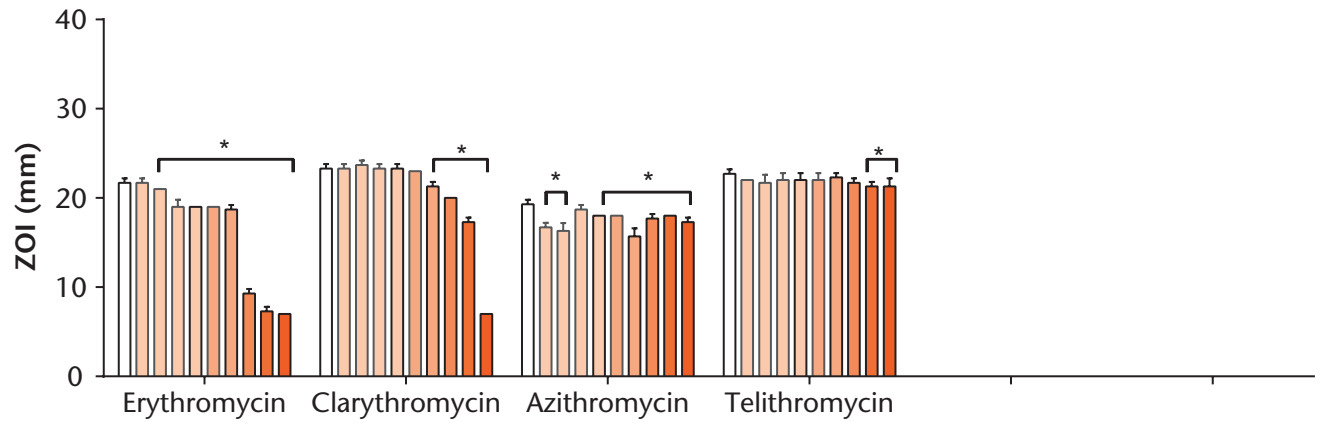

Macrolides and Ketolides

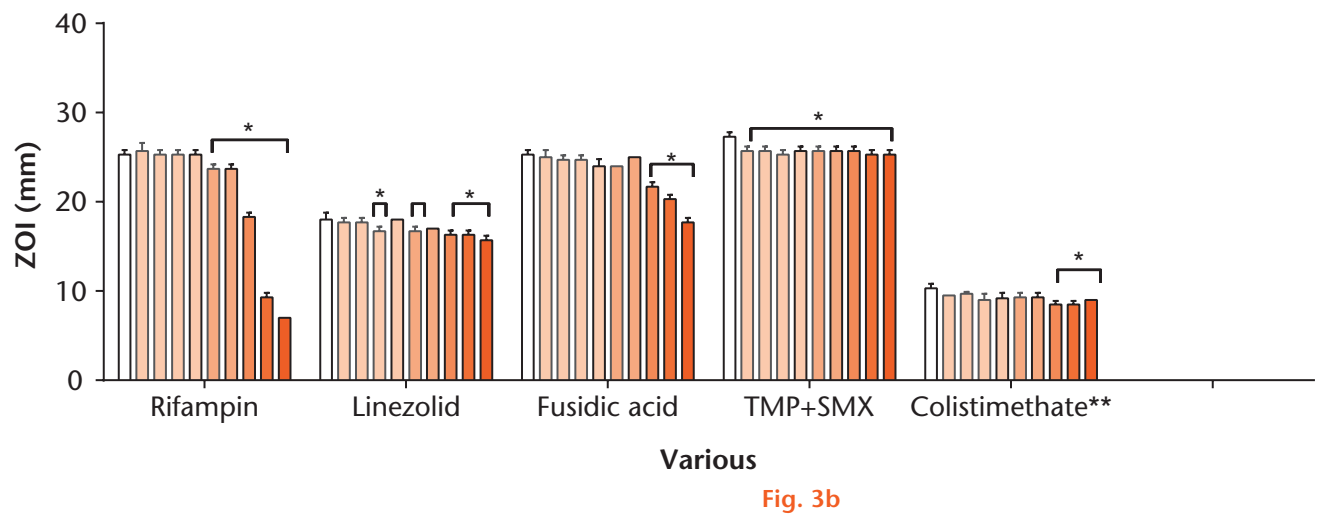

Charts showing thermal stability measured for each antibiotic over time, as determined by bioassay. Results are expressed as means from triplicate experiments. Zone of inhibition (ZOI) indicates diameter of the zone of inhibition, in millimetres. Statistically significant variations of the ZOI relative to starting values are marked with an asterisk where appropriate. Antibiotic solutions were prepared and maintained at $37^{\circ} \mathrm{C}$ for 42 days (body temperature group). Of note, temocillin and aztreonam have been tested with E. coli NCTC 12241, and colistimethate with P. aeruginosa PAO1, whereas all other results are from inhibition of S. aureus NCTC 12973. 
Table II. In vitro degradation half-lives, calculated from quantitative data by LC-MS/MS and FPIA as available from the body temperature group

\begin{tabular}{|c|c|}
\hline Antibiotic drugs & $t_{1 / 2} h$ (days) \\
\hline \multicolumn{2}{|l|}{ Penicillins } \\
\hline Amoxicillin & $150(6.3)$ \\
\hline Flucloxacillin & $381(15.9)$ \\
\hline Piperacillin & $410(17.1)$ \\
\hline Tazobactam & $619(25.8)$ \\
\hline \multicolumn{2}{|l|}{ Cephalosporins } \\
\hline Cefazolin & $265(11.0)$ \\
\hline Cefuroxime & $81(3.4)$ \\
\hline Ceftriaxone & $261(10.9)$ \\
\hline Ceftazidime & $176(7.3)$ \\
\hline Cefepime & $115(4.8)$ \\
\hline \multicolumn{2}{|c|}{ Carbapenems and monobactams } \\
\hline Imipenem & $72(3.0)$ \\
\hline Cilastatin & $3202(133.4)$ \\
\hline Meropenem & $67(2.8)$ \\
\hline Ertapenem & $96(4.0)$ \\
\hline \multicolumn{2}{|c|}{ Glycopeptides and lipopeptides } \\
\hline Vancomycin & Stable \\
\hline Daptomycin & $945(39.4)$ \\
\hline \multicolumn{2}{|l|}{ Aminoglycosides } \\
\hline Amikacin & Stable \\
\hline Gentamicin & Stable \\
\hline Tobramycin & Stable \\
\hline \multicolumn{2}{|l|}{ Quinolones } \\
\hline Ciprofloxacin & Stable \\
\hline Levofloxacin & Stable \\
\hline \multicolumn{2}{|l|}{ Various } \\
\hline Rifampicin & $95(4.0)$ \\
\hline Linezolid & Stable \\
\hline Colistimethate & Stable \\
\hline
\end{tabular}

$t_{1 / 2}$, half-life

There was no significant difference between samples subjected and those not subjected to the initial heat exposure. "Stable" indicates a degradation below the limit of detection, that is, the observed variability was smaller than the known analytical variability. After a degradation of about $25 \%$ induced by the initial short-term heat treatment, gentamicin remains stable (see Fig. 2). Cilastatin is a renal dehydropeptidase inhibitor co-administered to imipenem.

applications in other clinical settings wherein antibiotic stability is also an issue, such as administration of antibiotics by continuous infusion or via portable elastomeric pumps for parenteral outpatient treatment. ${ }^{11-16,21}$ The latter example certainly is an issue since portable devices might reach temperatures of up to $35^{\circ} \mathrm{C}$ under real-life conditions. $^{21}$

Overall, large differences in antibiotic stability have been observed, ranging from rapid degradation of antibiotics within one to two weeks, to almost complete stability over the entire six-week test period. Not unexpectedly, beta-lactam antibiotics were found to be almost completely degraded over time, with the exception of the monobactam aztreonam, which apparently has a higher stability. ${ }^{16,21,22}$ Some beta-lactams, namely flucloxacillin, ticarcillin with clavulanate, piperacillin with tazobactam, cefazolin and ceftriaxone, maintained relevant antibacterial activity for up to 21 days. The greater inhibition zones observed in the bioassays for the combination of amoxicillin with clavulanate, compared with amoxicillin alone, might be explained by a synergistic antibacterial effect of the combination. ${ }^{23,24}$ On the other hand, clavulanate is known to catalyse the degradation of amoxicillin in aqueous solution. ${ }^{25}$ However, this has not been quantified in our experiment. Temocillin has been reported to yield promising results for the treatment of outpatients using continuous perfusion pumps, ${ }^{13}$ but performed poorly in our study, showing a rapid decay of the antimicrobial activity measured by the Kirby-Bauer assay. Excellent long-term stability has been confirmed in our study for aminoglycosides and glycopeptides, strengthening their suitability and use in the orthopaedic setting. ${ }^{3-5,14,22}$ Tetracyclines and quinolones were highly thermo-stable according to our study but may not be acceptable for local application due to their reported cellular toxicity. ${ }^{26,27}$ Linezolid, trimethoprim with sulfamethoxazole, and, to a lesser extent, fusidic acid, all appeared to have good stability, without known relevant toxicity at the concentrations tested. ${ }^{27}$ Rifampin, while being a cornerstone in the treatment of implant-associated Staphylococci infections ${ }^{17}$ showed a rapid decrease of antibacterial activity after two weeks, as measured by bioassay.

Our investigations indicate that the initial short-term temperature elevation mimicking curing of PMMA has limited, if any, impact on antibiotic stability overall. Gentamicin was the only drug that was found to be affected to some extent, with a reduction of about $25 \%$ caused by the initial heat treatment. This was not observed for the other aminoglycosides tested. For vancomycin and colistimethate, significant discrepancies were observed in the heat group between the mass spectrometry quantification and bioassay. The prodrug colistimethate needs to be hydrolysed to yield the active colistin, and the extent of such a reaction in the disk diffusion test is uncertain, while the poor diffusion of the active colistin on agar also limits interpretability of the bioassay results, even if colistin resistance can be identified quite reliably with this method. ${ }^{28}$ For vancomycin, possible changes in the solution's matrix after the initial heat treatment may potentially diminish antibiotic bioavailability via alteration of the antibiotic adsorption onto the paper disk and diffusion, hence diminishing the antimicrobial activity.

The quantification of LC-MS/MS and FPIA provides precise drug concentrations, and allows us to determine the in vitro degradation half-lives (Table II). However, such methods were not available for all antibiotics, notably the macrolides/ketolides and the tetracyclines/glycylcyclines (Table I). Disk diffusion tests have been used for a qualitative, rather than quantitative, assessment of activity for these antibiotics. The establishment of calibration curves to enable semi-quantitative measurement of such a large number of antibiotics would have been technically challenging, relatively imprecise, and interpretation would have been limited to narrow ranges of concentrations. ${ }^{29}$ Besides, for glycopeptides and daptomycin, the disk 
diffusion approach can be used only for ascertaining the presence of antibacterial activity. ${ }^{20,30}$ More generally, the comparison with recommended breakpoints for susceptibility testing using bioassays is also limited since, for most antibiotics in our experiment, quantities that have to be loaded onto paper disks would exceed the starting concentration, which had been defined in consideration of local toxicity issues. ${ }^{19,20,27,30}$

A strength of this study is that two different analytical approaches have been used. Precise quantification of the most commonly prescribed antibiotic agents has been performed by LC-MS/MS or FPIA, while antibacterial activity has been ascertained for all antibiotics by disk diffusion assays. Overall, the bioassays were generally congruent with the quantitative analysis by LC-MS/MS or FPIA. The LC-MS/MS method reveals that gentamicin was in part degraded by the initial heat treatment, while this $25 \%$ drop in concentration could not be detected by the less sensitive disk diffusion assay. Thus, tobramycin might be a more appropriate antibiotic to associate with PMMA, as it has an equivalent spectrum of activity ${ }^{31}$ but improved stability in our experiment. Of course, tissue penetration is an additional factor in the performance of any antibiotic, be it locally or systemically applied, even if difficult to interpret. $^{32,33}$ Data on antibiotic penetration of tobramycin versus gentamicin are limited, ${ }^{32}$ but the expectation would be that it would be similar due to very similar physicochemical properties. ${ }^{34}$ Even if all assays had been performed as a batch at the end of the experiment, no degradation of the antibiotics during storage would be expected, even for the more fragile beta-lactams, as the samples have been kept continuously at $-80^{\circ} \mathrm{C} .35$

Limitations of our experimental setting have to be acknowledged, particularly regarding the heat exposure mimicking the exothermic process taking place during the curing of bone cement. The temperature profile recorded in our study is specific to a given cement and form. Cements with other specifications and other quantities and forms may yield different temperature-time profiles. $^{36}$ The experimental design, i.e. subjecting an aqueous solution to a steam autoclave, may not have reproduced a temperature-time profile that strictly followed the one observed during the solid-state cement curing as boiling of the test solution had to be avoided. The use of pressure-resistant closed containers might allow testing of the potential effect of higher temperatures, but these were not available for the required volumes. While no discrepancies were observed between LC-MS/MS or FPIA and disk diffusion tests, disk diffusion data are available for more time points within the study period, while quantification assays suffer from a gap between day seven and day 42 . The schedule had been chosen to adapt best to exponential degradation of unstable drugs, while still being able to describe longterm stability. The results, however, do not indicate that a certain degradation profile would not have been detected. Another issue could be the fact that a number of antibiotics were tested in combination, however, the effects of these combinations cannot be evaluated as the individual drugs were not tested in isolation. Last, but not least, the test samples were solutions of antibiotics, also containing the various components of the pharmaceutical preparations such as preservatives, buffers and stabilising agents that reflect only partially the interactions with biological solutions and the various reactions occurring during cement curing.

In conclusion, our study provides information of interest not only for local application of antibiotics in orthopaedic and trauma surgery, but also in other medical specialties that require prolonged antibiotic exposure and for which drug stability is also an issue. In order to maximise the applicability of our data, we did not test elution from any material, or measure degradation at room or refrigerated temperatures. Our experiments have allowed the identification of classes of antibiotics or specific agents deemed useful for further investigation because of favourable in vitro stability. Additionally, this dataset is relevant for the next generation of antibiotic carriers as it provides data pertinent to the thermal stability of a range of antibiotics that may be considered for this use. Nevertheless, any future studies on antibiotic carriers should include careful assessment of antibiotic release kinetics, both in vitro and in vivo.

\section{References}

1. Hanssen AD, SpangehI MJ. Practical applications of antibiotic-loaded bone cement for treatment of infected joint replacements. Clin Orthop Relat Res 2004;427:79-85.

2. Henry SL, Galloway KP. Local antibacterial therapy for the management of orthopaedic infections. Pharmacokinetic considerations. Clin Pharmacokinet 1995; 291:36-45

3. Iarikov D, Demian H, Rubin D, Alexander J, Nambiar S. Choice and doses of antibacterial agents for cement spacers in treatment of prosthetic joint infections: review of published studies. Clin Infect Dis 2012;55:1474-1480.

4. Cui O, Mihalko WM, Shields JS, Ries M, Saleh KJ. Antibiotic-impregnated cement spacers for the treatment of infection associated with total hip or knee arthroplasty. J Bone Joint Surg [Am]2007;89-A:871-882.

5. Kanellakopoulou K, Giamarellos-Bourboulis EJ. Carrier systems for the local delivery of antibiotics in bone infections. Drugs 2000;59:1223-1232.

6. Anagnostakos K, Kelm J. Enhancement of antibiotic elution from acrylic bone cement. J Biomed Mater Res B App/ Biomater 2009;90:467-475

7. Anagnostakos K, Schroder K. Antibiotic-impregnated bone grafts in orthopaedic and trauma surgery: a systematic review of the literature. Int J Biomater 2012:538061.

8. Swieringa AJ, Goosen JH, Jansman FG, Tulp NJ. In vivo pharmacokinetics of a gentamicin-loaded collagen sponge in acute periprosthetic infection: serum values in 19 patients. Acta Orthop 2008;79:637-642.

9. Wahl P, Livio F, Jacobi M, Gautier E, Buclin T. Systemic exposure to tobramycin after local antibiotic treatment with calcium sulphate as carrier material. Arch Orthop Trauma Surg 2011;131:657-662.

10. Mousset B, Benoit MA, Delloye C, Bouillet R, Gillard J. Biodegradable implants for potential use in bone infection. An in vitro study of antibiotic-loaded calcium sulphate. Int Orthop 1995;19:157-161.

11. Arlicot N, Marie A, Cade C, Laffon M, Antier D. Stability of amoxicillin in portable pumps is drug concentration dependent. Pharmazie 2011;66:631-632.

12. Berthoin K, Le Duff CS, Marchand-Brynaert J, Carryn S, Tulkens PM. Stability of meropenem and doripenem solutions for administration by continuous infusion. J Antimicrob Chemother 2010;65:1073-1075. 
13. De Jongh $\mathbf{R}$, Hens $\mathbf{R}$, Basma $\mathbf{V}$, et al. Continuous versus intermittent infusion of temocillin, a directed spectrum penicillin for intensive care patients with nosocomial pneumonia: stability, compatibility, population pharmacokinetic studies and breakpoint selection. J Antimicrob Chemother 2008;61:382-388.

14. Raverdy V, Ampe E, Hecq JD, Tulkens PM. Stability and compatibility of vancomycin for administration by continuous infusion. J Antimicrob Chemother 2013;68:1179-1182.

15. Vella-Brincat JW, Begg EJ, Gallagher K, et al. Stability of benzylpenicillin during continuous home intravenous therapy. J Antimicrob Chemother 2004;53:675-677.

16. Viaene E, Chanteux H, Servais H, Mingeot-Leclercq MP, Tulkens PM. Comparative stability studies of antipseudomonal beta-lactams for potential administration through portable elastomeric pumps (home therapy for cystic fibrosis patients) and motor-operated syringes (intensive care units). Antimicrob Agents Chemother 2002; 46:2327-2332.

17. Osmon DR, Berbari EF, Berendt AR, et al. Infectious Diseases Society of America. Diagnosis and management of prosthetic joint infection: clinical practice guidelines by the Infectious Diseases Society of America. Clin Infect Dis 2013;56:e1-e25.

18. Stengel D, Bauwens K, Sehouli J, Ekkernkamp A, Porzsolt F. Systematic review and meta-analysis of antibiotic therapy for bone and joint infections. Lancet Infect Dis 2001;1:175-188

19. Matuschek E, Brown DF, Kahlmeter G. Development of the EUCAST disk diffusion antimicrobial susceptibility testing method and its implementation in routine microbiology laboratories. Clin Microbiol Infect 2014;20:0255-0266.

20. No authors listed. EUCAST. Breakpoint tables for interpretation of MICs and zone diameters version 5, 2015. http://www.eucast.org/ast_of_bacteria/previous_ versions_of_documents/ (date last accessed 1 March 2017)

21. Arlicot N, Rochefort GY, Schlecht D, et al. Stability of antibiotics in portable pumps used for bronchial superinfection: guidelines for prescribers. Pediatrics 2007;120:1255-1259.

22. Hsieh PH, Chang YH, Chen SH, Ueng SW, Shih CH. High concentration and bioactivity of vancomycin and aztreonam eluted from Simplex cement spacers in twostage revision of infected hip implants: a study of 46 patients at an average follow-up of 107 days. J Orthop Res 2006:24:1615-1621.

23. Finlay J, Miller L, Poupard JA. A review of the antimicrobial activity of clavulanate. J Antimicrob Chemother 2003;52:18-23.

24. Sánchez Navarro A. New formulations of amoxicillin/clavulanic acid: a pharmacokinetic and pharmacodynamic review. Clin Pharmacokinet 2005;44: 1097-1115.

25. Vahdat L, Sunderland B. The influence of potassium clavulanate on the rate of amoxicillin sodium degradation in phosphate and acetate buffers in the liquid state. Drug Dev Ind Pharm 2009;35:471-479.

26. Holtom PD, Pavkovic SA, Bravos PD, et al. Inhibitory effects of the quinolone antibiotics trovafloxacin, ciprofloxacin, and levofloxacin on osteoblastic cells in vitro. J Orthop Res 2000;18:721-727.

27. Rathbone CR, Cross JD, Brown KV, Murray CK, Wenke JC. Effect of various concentrations of antibiotics on osteogenic cell viability and activity. J Orthop Res 2011;29:1070-1074.

28. Galani I, Kontopidou F, Souli M, et al. Colistin susceptibility testing by Etest and disk diffusion methods. Int J Antimicrob Agents 2008;31:434-439.
29. Bonev B, Hooper J, Parisot J. Principles of assessing bacterial susceptibility to antibiotics using the agar diffusion method. I Antimicrob Chemother 2008;61:1295-1301.

30. No authors listed. CLSI. Performance standards for antimicrobial susceptibility testing. M100-S25. Clinical and Laboratory Standards Institute, January 2015. http://shop.clsi.org/site/Sample_pdf/M100S25_sample.pdf (date last accessed 24 March 2017).

31. Hanberger H, Edlund C, Furebring M, et al. Swedish Reference Group for Antibiotics. Rational use of aminoglycosides-review and recommendations by the Swedish Reference Group for Antibiotics (SRGA). Scand J Infect Dis 2013:45:161-175.

32. Landersdorfer CB, Bulitta JB, Kinzig M, Holzgrabe U, Sörgel F. Penetration of antibacterials into bone: pharmacokinetic, pharmacodynamic and bioanalytical considerations. Clin Pharmacokinet 2009;48:89-124.

33. Mouton JW, Theuretzbacher U, Craig WA, et al. Tissue concentrations: do we ever learn? J Antimicrob Chemother 2008;61:235-237.

34. Krause KM, Serio AW, Kane TR, Connolly LE. Aminoglycosides: An Overview. Cold Spring Harb Perspect Med 2016;6:a027029.

35. Zander J, Maier B, Zoller M, et al. Effects of biobanking conditions on six antibiotic substances in human serum assessed by a novel evaluation protocol. Clin Chem Lab Med 2016;54:265-274.

36. Sih GC, Connelly GM, Berman AT. The effect of thickness and pressure on the curing of PMMA bone cement for the total hip joint replacement. J Biomech 1980;13:347-352.

Acknowledgments

Dieter Wahl and Ladina Fliri from AO Research Institute Davos are thanked for expert assistance during temperature measurements.

Funding Statement

- Funding of this study has been supported by AO Trauma as part of the Clinical Priority Program Bone Infection, and also by the OrthoTrauma Foundation. The Swiss National Science Foundation (SNF REQUIP Grant N 326000-121314/1) and a matching fund from the University of Lausanne-Lausanne University Hospita (UNILCHUV) have made possible the acquisition of the HPLC-MS/MS instrumentation.

Author Contribution

E. Samara: Establishment of protocol, literature search, data collection, writing of manuscript.

- T. F. Moriarty: Study design, establishment of protocol, statistical analysis, writing of manuscript.

L. A. Decosterd: Establishment of protocol, data collection, statistical analysis, writing of manuscript.

R. G. Richards: Financial and legal coordination, review of project design, review of the manuscript.

E. Gautier: Financial coordination, review of project design, review of the manuscript. P. Wahl: Study design, establishment of protocol, literature search, statistical analy sis, writing of manuscript.

ICMJE Conflicts of Interest

None declared.

(c) 2017 Moriarty et al. This is an open-access article distributed under the terms of the Creative Commons Attributions licence (CC-BY-NC), which permits unrestricted use, distribution, and reproduction in any medium, but not for commercial gain, provided the original author and source are credited. 